\title{
On the Compressibility Properties of the Wine-Rack like Carbon Allotropes and Related Poly(phenylacetylene) Systems
}

Edera P. Degabriele ${ }^{1}$, Daphne Attard ${ }^{1}$, James N. Grima-Cornish ${ }^{2}$, Roberto Caruana-Gauci ${ }^{1}$, Ruben Gatt ${ }^{1}$, Kenneth E. Evans ${ }^{3}$ and Joseph N. Grima*1,2

${ }^{1}$ Metamaterials Unit, Faculty of Science, University of Malta, Msida MSD 2080, Malta.

${ }^{2}$ Department of Chemistry, Faculty of Science, University of Malta, Msida MSD 2080, Malta.

${ }^{3}$ College of Engineering, Mathematics and Physical Sciences, University of Exeter, Exeter EX4 4QF, UK.

\begin{abstract}
Poly(phenylacetylene) sheets that mimic the geometry of a wine-rack-like structure have been predicted to exhibit negative Poisson's ratios off-axis. However, their potential to exhibit negative linear compressibility (NLC) has remained largely unexplored. In this work, the compressibility and other mechanical properties of wine-rack-like poly(phenylacetylene) networks with 1,2,4,5 tetra-substituted phenyls as well as their equivalent with allene or cyclobutadiene centres are simulated to assess their ability to exhibit negative linear compressibility on-axis and off-axis. It is shown that some of these systems can indeed exhibit negative linear compressibility whist others exhibit a near-zero compressibility. The results are compared to the compressibility properties of other poly(phenylacetylene) networks reported in literature as well as with those predicted from the analytical model for an idealised wine-rack structure deforming through hinging. Results suggest that these mechanical properties are arising from a wine-rack-like mechanism, and there is a good agreement with the theoretical model, especially for systems with longer acetylene chains whose geometry is closer to that of the idealised wine-rack.
\end{abstract}

\section{Introduction}

In the last decades, there has been a growing interest on hyper-conjugated carbon-based molecular networks in view of their very interesting topologies and the properties they afford $^{1-29}$. In particular, the literature describing materials with a negative Poisson's ratio (NPR, auxetic) ${ }^{12}$ is rich with studies on the structure and mechanical properties of poly(phenylacetylene) crystalline networks of various forms and connectivity ${ }^{14,19,20,22,30}$ which compliments the ever-growing body of knowledge in this field ${ }^{12,14-16,31-50}$. Studies 
which focus on the Poisson's ratio of such materials include work on flexynes and reflexynes, where the phenyl ring is tri-substituted ${ }^{12,14,21,22}$; work on triangles and wine-rack like structures, where the phenyl ring is tetra-substituted ${ }^{20,22,31}$; as well as penta- and hexasubstituted graphyne, graphydiyne and related systems ${ }^{6,9,19,20,31}$. Most of these papers have focused on the variation of the Poisson's ratio with various structural parameters, such as length of the acetylene chain, manner of substitution and the direction of uniaxial stretching. More recent studies on mechanical metamaterials ${ }^{51}$ have started to give more importance to the compressibility of such systems, that is, the variation in dimensions that occur when they are subjected to externally applied hydrostatic pressure ${ }^{52-63}$. Although some of the aforementioned systems have the amenable geometric requirements (e.g. a wine-rack-like geometry) to permit one to infer that they may exhibit negative linear compressibility (NLC), to this date these crystalline networks have not been formally analysed vis-à-vis their compressibility properties. In view of this, this paper attempts to address this lacuna by reporting and analysing the compressibility and other mechanical properties of poly(phenylacetylene) networks with 1,2,4,5 tetra-substituted phenyls and two crystalline graphite-like layered carbon allotropes together with a re-analysis of the properties of the crystalline equivalents of graphyne, graphdiyne and other related systems which have comparable characteristics.

\section{Methodology}

\section{(a) Systems Studied}

The systems shown in Figure 1 and their homologues with longer chains having $n \in\{1,2,3,4,5\}$ triple bonds in their acetylene chains were considered in this study. The systems shown in (a) and (b) are carbon allotropes, which have geometric features that are archetypal of the wine-rack model (see Figure 2) where acetylene chains are connected via either an allene or a cyclobutadiene centre henceforth referred to as C2- $n$ and C4- $n$ systems where $n$ is the number of triple bonds in the acetylene chain. Similar to these are the poly(phenylacetylene) systems, represented in (c), where the allene is replaced by a 1,2,4,5susbtituted phenyl centre, referred to as C6- $n$ systems. The systems in (d) to (g) are other poly(phenylacetylene) systems with different connectivities, as discussed in Degabriele et al. and works cited therein. These are ' $1,2,3,4$ ' tetra-substituted, ' $1,2,3,4,5,6$ ' hexa-substituted, 
'1,2,3,4,5' penta-substituted and '1,3,5' tri-substituted phenyl centres, which are also known as 'polytriangles' (Fig. 1d), 'graphyne, graphdiyne, etc.' (Fig. 1e), 'megaflexynes' (Fig. 1f) and 'flexynes' (Fig. 1g)' respectively. For this present study, all systems were considered as crystals with different layers being oriented in the (100) plane and able to stack freely in the [100] direction.

\section{INSERT FIGURE 1}

\section{INSERT FIGURE 2}

\section{(b) Simulations}

The systems in Fig. 1(a) and (b) with $n=1,2, \ldots, 5$ were constructed within the molecular modelling package Cerius ${ }^{2}$ as crystalline systems with the unit cells as shown in Figure 1. All the crystals were oriented with respect to the Cartesian system in such a way that the crystal [001] direction lies parallel to the global z-axis and the [010] crystal direction is aligned in the global yz-plane with the [100] left to orient freely in any direction. This method of construction is similar to that described in other studies ${ }^{20}$ and allows the layers to remain well oriented in the $y z$-plane during the simulation so as to facilitate data comparison and interpretation.

The energy expression was set up using the PCFF force-field ${ }^{64,65}$ as implemented in Cerius ${ }^{2}$. Default settings were used, with the exception that non-bond interactions were summed up using the Ewald summation technique ${ }^{66}$. The systems were then optimised so as to minimise the energy of the system to default Cersius ${ }^{2}$ high convergence criteria which include an atomic RMS gradient less than $0.001 \mathrm{kcal} \mathrm{mol}^{-1} \AA^{-1}$. Following this, the full $6 \times 6$ stiffness matrix $\mathbf{C}$ and its inverse, the compliance matrix $\mathbf{S}$, were simulated using the second derivative method since:

$$
c_{i j}=\frac{1}{V} \frac{\partial^{2} E}{\partial \varepsilon_{i} \partial \varepsilon_{j}}, \quad i, j=1,2, \ldots, 6
$$

where V is the volume of the unit cell, E represents the energy expression and ei $(i=1,2, \ldots 6)$ are the strain components where $1=x x, 2=y y, \ldots, 6=z y$.

The equivalent optimised systems as well as the respective stiffness and compliance matrices of the systems shown in Fig. 1(c)-(g) are taken from Degabriele et al. ${ }^{20}$. 


\section{(c) Calculation of compressibility and other properties}

The linear compressibilities, Poisson's ratios and moduli in the (100) yz-plane were calculated from the transformed compliances, $s_{i j}(\zeta)$, which correspond to the compliance matrix of the crystal after the crystal is re-oriented following rotation by an angle $\zeta$ around the $x$-axis as follows:

$$
\begin{array}{ll}
\text { Compressibility: } & \beta_{y}(\zeta)=s_{21}(\zeta)+s_{22}(\zeta)+s_{23}(\zeta) \\
\text { Poisson's ratio: } & v_{y z}(\zeta)=-\frac{s_{32}(\zeta)}{s_{22}(\zeta)} \\
\text { Moduli: } & E_{y}(\zeta)=\frac{1}{s_{22}(\zeta)}, \quad G_{y z}(\zeta)=\frac{1}{s_{44}(\zeta)}
\end{array}
$$

\section{Results and Discussion}

The results of simulations are shown in Figure 3 - 4 and in the Supplementary information. In particular, Figure 3 shows images of the $y z$-projection of the energy minimised systems with the wine-rack like geometries having (a) allene (b) cyclobutadiene and (c) phenyl central units where $n=5$, together with the corresponding plots of the linear compressibility, Poisson's ratio and moduli. (See Supplementary Information Figures S1 - S3 for images of all the minimised systems.) Note that the data for (a) and (b) is being reported for the first time whilst that of (c) is a re-analysis of the data published by Degabriele et al. From this data it is evident that whilst the systems with the allene and phenyl centres behave in a highly comparable manner and exhibit, in different directions, both NLC and NPR, the system with a cyclobutadiene centre exhibits different trends with the Poisson's ratio being always positive and the compressibility being near zero. Reported in Figure 4 are the compressibility properties of the other poly(phenylacetyelene) systems which all exhibit quasi-in-plane isotropic positive compressibilities.

\section{INSERT FIGURE 3}

\section{INSERT FIGURE 4}

This compressibility, Poisson's ratio and stiffness behaviour may be explained by looking at the equivalent highly idealised wine-rack like models which deform exclusively through 
changes in angles between the ligaments ${ }^{61}$, as illustrated in Figure 2. Recognising that the systems studied here may be, to a very rough approximation, be considered in terms of a system made from ligaments of length $l$, which are connected together through hinges to make a wine-rack like structure with either $\theta=120^{\circ}$ or $90^{\circ}$, see Figure 5 , then the equations for the compressibility, Poisson's ratio and moduli in the plane of the structures for loading at angle $\zeta$ to the $O x_{1}$-axis may be expressed as:

(a) For the systems $\theta=120^{\circ}$ :

Compressibility: $\quad \beta_{1}(\zeta)=\frac{l^{2} z}{8 \sqrt{3} K_{h}}(1+2 \cos (2 \zeta))$

Poisson's Ratios: $v_{12}(\zeta)=\frac{1-3 \tan ^{2}(\zeta)}{3-\tan ^{2}(\zeta)}$

Moduli: $\quad E_{1}(\zeta)=\frac{16 \sqrt{3} K_{h}}{l^{2} z} \frac{1}{(1+2 \cos (2 \zeta))^{2}}, G_{12}(\zeta)=\frac{K_{h} \sqrt{3}}{l^{2}} \frac{1}{\sin ^{2}(2 \zeta)}$

(b) For the systems $\theta=90^{\circ}$ :

Compressibility: $\quad \beta_{1}(\zeta)=0$

Poisson's Ratios: $\quad v_{12}(\zeta)=1$

Moduli: $\quad E_{1}(\zeta)=\frac{8 K_{h}}{l^{2} z} \frac{1}{\cos ^{2}(2 \zeta)}, G_{12}(\zeta)=\frac{2 K_{h}}{l^{2}} \frac{1}{\sin ^{2}(2 \zeta)}$

where $K_{h}$ is the stiffness constant of the hinges and $z$ is their out-of-plane thickness. It is interesting to note that despite the many differences between the molecular-level systems and the idealised 'wine rack' model, including the lack of a 'mechanical hinge', these equations for the hinging model can satisfactorily predict most of the properties demonstrated by the nanoscale systems thus suggesting that a wine-rack like mechanism may indeed be operating. Note that in the present work the $\mathrm{O} x_{1}$ and $\mathrm{O} x_{2}$ axis in the analytical model correspond to the global $y$ - and $z$ - direction i.e. the [001] crystal axis (permanently aligned with the $z$-axis) corresponds to the $\mathrm{O} x_{2}$ direction.

INSERT FIGURE 5 
In particular, the analytical model with $\theta=120^{\circ}$ predicts the highest Young's moduli, tending to infinity, off-axis when $\zeta= \pm 60^{\circ}$ i.e. directions which correspond to the directions of the ligament. This behaviour occurs because the analytical model assumes that the ligaments are perfectly rigid i.e. the only mode of deformation is through relative rotation of the ligaments (hinging). When the loading direction is parallel to the ligaments, these can no longer rotate thus rendering the structure 'undeformable'. This behaviour is replicated, to some extent, by the C2, C4 and C6 systems where the same trends are reproduced i.e. for $\zeta \in\left[-90^{\circ},+90^{\circ}\right]$ the Young's modulus assumes very low values on-axis and large values at off-axis angles close to (a) $\zeta= \pm 60^{\circ}$, (b) $\zeta= \pm 45^{\circ}$ and (c) $\zeta= \pm 60^{\circ}$ respectively, which angles correspond to the direction of the acetylene chains. Obviously, in the case of the molecular systems, infinite Young's modulus is not observed since in these more realistic systems, alternative modes of deformation can be manifested. A particularly interesting observation is that whilst in the case of the phenyl-centred C6 systems maximum Young's moduli occur at almost exactly $\zeta= \pm 60^{\circ}$ off-axis, in the case of the allene, these maximum moduli occur at slightly different values. For example as noted in Table 1 , for $n=5$, maximum Young's modulus is at $\zeta= \pm 56.91^{\circ}$ for $\mathrm{C} 2$ and at $\zeta= \pm 59.74^{\circ}$ for C6. This may be explained by the fact that, as shown in Figure 5, in the case of the phenyl-centred C6 systems a superimposed wine-rack model would have its ligaments parallel to the acetylene chains and meeting at the centre of the phenyl rings at an angle of $60^{\circ} / 120^{\circ}$ to each other given that the phenyl link has an amenable geometry which permits such angles. However in the case of the C2 geometry, a superimposed wine-rack model would not have its ligaments perfectly parallel to the acetylene chains since in this case, the hypothetical ligaments which would form a wine-rack would pass through the centre of the double bond and through the centres of the acetylene chains. Note that as the length of the acetylene chain increases, this effect becomes less pronounced and the angles tend to the idealised values of $\zeta= \pm 60^{\circ}$. In the case of C4, maximum Young’s moduli always occur at $c . \pm 45^{\circ}$ (see Table 1 ).

\section{INSERT TABLE 1}

\section{INSERT FIGURE 5}

The Poisson's ratios afforded by the C2, C4 and C6 systems are also in agreement with some of the predictions from the analytical model. For example, for $\zeta \in\left[-90^{\circ},+90^{\circ}\right]$, the 
analytical model predicts that at $\theta=120^{\circ}$ negative Poisson's ratios are given when $\zeta$ is in the range of $30^{\circ}<\zeta<60^{\circ}$ and $-60^{\circ}<\zeta<-30^{\circ}$. This is in good agreement with the off-axis plots given in Figure 3 where, for example, the C2-5 and C6-5 shows auxetic behaviour in the range of $34^{\circ}<\zeta<56^{\circ}$ and $-34^{\circ}<\zeta<-56^{\circ}$ for $\mathrm{C} 2-5$, and, $33^{\circ}<\zeta<60^{\circ}$ and $-28^{\circ}<\zeta<-59^{\circ}$ for C6-5. In the case of C4 systems when $\theta=90^{\circ}$, the analytical model correctly predicts the Poisson's ratios of $c .+1$ for $v_{\mathrm{yz}}$ and $v_{\mathrm{zy}}$ for the molecular systems with longer acetylene chains but is unable to predict the anisotropy in this property since the idealised model predicts a Poisson's ratio of +1 for all values of $\zeta$ and ligament lengths. Instead, the mechanical properties of the C4 systems show that the on-axis Poisson's ratio approximates +1 as the length of the acetylene chain increases but is not isotropic in-plane as this decreases as $\zeta \rightarrow \pm 45^{\circ}$. In other words, the Poisson's ratio is dependent on both the length of the acetylene chain and direction of loading, two features which were not predicted by the analytical model. This is because in these nanoscale systems, which lack proper welldefined hinges at the joints, it is unreasonable to assume 'hinging' to be the exclusive operating mechanism. Instead, the overall behaviour of the system would be the result of the interplay of a number of deformation mechanisms, which may be expressed in changes in the bond lengths and bond angles, and the Poisson's ratio is reflective of all these deformation mechanisms.

In terms of the compressibility, for $\zeta \in\left[-90^{\circ},+90^{\circ}\right]$, the systems presented in Figure 2 (a) and (c) show negative compressibility of considerable magnitude when $\zeta>57^{\circ}$ and $-57^{\circ}<\zeta$ respectively. This is in good agreement with the range of $\zeta>60^{\circ}$ and $\zeta<-60^{\circ}$ predicted by the analytical model. In this case, as with other properties, the best agreement between the analytical model predictions and the simulated properties for the molecular systems are exhibited for the systems with the longer acetylene chains, a feature which may be explained through the observation that the systems with longer acetylene chains bear a closer geometric resemblance to their equivalent hypothetical wine-rack model. It is also interesting to note that the analytical model correctly predicts the very low magnitude of the compressibility of the C4 systems, which are an order of magnitude lower than their corresponding C2 and C6 systems.

In an attempt to obtain more information on the nanoscale ons of these systems when subjected to pressure or uniaxial loads, a series of additional simulations meant to simulate 
the behaviour of the system under hydrostatic pressure or uniaxial stresses were carried out on the C2-5 and C6-5 systems, details of which are provided in the Supplementary Information (Figure S5 and S6). The simulations suggest that under a hydrostatic pressure, all systems contract along the $x$-direction (positive linear compressibility) so that the poly(phenylacetylene) layers are forced closer to each other. Moreover, as illustrated in Figure 6, these simulations confirm that in the yz-plane a wine-rack-like mechanism is indeed operational in all of these systems. In fact, in all systems studied the ligaments deform in a manner which make them appear as if they are rotating relative to each other in a scissor-like manner. On closer inspection, one may note that this rotation is caused by both changes in the angles between the acetylene chains and the allene/cyclobutadiene/phenyl group (which would correspond to hinging in the analytical model) and changes in the angles along the acetylene chain (which would correspond to flexure). Such behaviour where hinging and flexing occur simultaneously in an additive manner is concordant with what was predicted by Evans and Masters ${ }^{67}$ in their work on honeycombs and had also been observed when studying the flexyne/re-flexyne systems ${ }^{14}$. Also worth noting is that models by Barnes et al ${ }^{68}$ suggest that models involving 'flexing wine-rack-like' structures are not dissimilar to the models deforming through hinging such as those presented by Grima et $a l^{53}$ and Zhou and Zhang. ${ }^{69}$

\section{INSERT FIGURE 6}

At this point it is interesting to compare the behaviour of these three wine-rack like systems, in particular their linear compressibility properties, with the other poly(phenylacetylene) networks discussed by Degabriele et al. ${ }^{20}$ and works cited therein. If one looks at the compressibility values of the poly(phenylacetylene) networks shown in Figure 4, as noted above, one would note that all of them exhibit quasi-isotropic compressibility properties in the plane of the networks. This may be explained by the fact that the systems belong to a symmetry group which renders them isotropic in-plane. It is also interesting to note that the 1,2,3,4-substituted systems are characterised by much higher compressibility properties (i.e. respond to a greater extent when subjected to pressure); a feature which may be explained by the fact that these systems may easily be deformed through what may be described as relative rotations of the triangular unit ${ }^{70}$. In contrast both the 1,2,3,4,5 and 1,2,3,4,5,6 systems, shown in Figure 4, are very difficult to compress in-plane due to the fact that in such systems the triangular units 'lock' the systems and make them practically incompressible. ${ }^{71}$ 
Before concluding, it is important to appreciate some of the limitations of this work. First and foremost, the results being reported here are obtained solely from simulations, and have not yet been backed up by experimental findings. An experimental verification of what is being reported here is not expected to be a trivial process as the systems discussed have never yet been synthesised and characterised. However, it is encouraging that in recent years,

there has been considerable work which has looked at some of these systems ${ }^{4-11,23,24,27-29}$ and it is hence hoped that the present work will provide further impetus for experimentalists and theoreticians to further study these systems and characterise them. Also the discussion has focused on the in-plane behaviour of these systems, despite the fact that these crystalline systems are of a three-dimensional nature. In particular it should be noted that a hydrostatic pressure is applied in all directions and apart from the compressibility expansions in plane, there is also a considerable contraction which occurs out of plane as the separation between the adjacent layer decreases, despite the fact that the ligaments are deforming out of plane. This, together with the behaviour under shear loading, merits further investigation. It should also be appreciated that the work by Tretiakov et al. has also studied what can be considered as very similar structures to what was studied but using a rather different intermolecular potential. This work by Tretiakov et al. had suggested completely different properties, thus highlighting the critical role of the intermolecular potential on the macroscopic properties of the systems studied. ${ }^{72}$ Finally, it should be mentioned that the systems studied here are extensively delocalised, therefore resulting in them being highly conductive. This feature makes these systems potentially usable in a number of possible niche applications requiring this property together with the anomalous mechanical behaviour reported here. This feature should be studied further through the use of simulation methods which permit an accurate representation of the electronic state of the systems.

\section{Conclusion:}

This work has shown that it is possible to design graphite-like nano-systems which can exhibit unusual mechanical properties through mimicking of mechanisms occurring at the macroscale. This re-highlights the very strong dependence between the macroscopic mechanical properties and the geometric features which characterise the various nano- 
networks. It also shows that it is possible to design and engineer crystals which can be tailormade to exhibit particular combinations of mechanical properties; which may include NLC, near-zero compressibility and/or NPR.

\section{Acknowledgments:}

JNG acknowledges the support of the University of Malta research grant. The research work disclosed in this publication is funded by the ENDEAVOUR Scholarship Scheme (Malta). The scholarship may be part- human capital to create more opportunities and promote the wellbeing of Operational Programme II - Cohesion Policy 2014-2020, “Investing in financed by the European Union - European Social Fund (ESF) under society.” 


\section{References:}

(1) Hoffmann, R.; Hughbanks, T.; Kertesz, M.; Bird, P. H. Hypothetical Metallic Allotrope of Carbon. J. Am. Chem. Soc. 1983, 105, 4831-4832.

(2) Prasad, D. L. V. K.; Gerovac, N. M.; Bucknum, M. J.; Hoffmann, R. Squaroglitter: A 3,4-Connected Carbon Net. J. Chem. Theory Comput. 2013, 9, 3855-3859.

(3) Bu, H.; Zhao, M.; Dong, W.; Lu, S.; Wang, X. A Metallic Carbon Allotrope with Superhardness: A First-Principles Prediction. J. Mater. Chem. C 2014, 2, 2751-2757.

(4) Hu, M.; He, J.; Wang, Q.; Huang, Q.; Yu, D.; Tian, Y.; Xu, B. Covalent-Bonded Graphyne Polymers with High Hardness. J. Superhard Mater. 2014, 36, 257-269.

(5) Hu, M.; Shu, Y.; Cui, L.; Xu, B.; Yu, D.; He, J. Theoretical Two-Atom Thick Semiconducting Carbon Sheet. Phys. Chem. Chem. Phys. 2014, 16, 18118-18123.

(6) Cranford, S. W.; Buehler, M. J. Mechanical Properties of Graphyne. Carbon N. Y. 2011, 49, 4111-4121.

(7) Yue, Q.; Chang, S.; Kang, J.; Tan, J.; Qin, S.; Li, J. Magnetic and Electronic Properties of Alpha-Graphyne Nanoribbons. J. Chem. Phys. 2012, 136.

(8) Wang, S.; Si, Y.; Yuan, J.; Yang, B.; Chen, H. Tunable Thermal Transport and Mechanical Properties of Graphyne Heterojunctions. Phys. Chem. Chem. Phys. 2016, 18, 24210-24218.

(9) Peng, Q.; Ji, W.; De, S. Mechanical Properties of Graphyne Monolayers: A FirstPrinciples Study. Phys. Chem. Chem. Phys. 2012, 14, 13385-13391.

(10) Xiao, J.; Shi, J.; Liu, H.; Xu, Y.; Lv, S.; Luo, Y.; Li, D.; Meng, Q.; Li, Y. Efficient CH3NH3PbI3 Perovskite Solar Cells Based on Graphdiyne (GD)-Modified P3HT Hole-Transporting Material. Adv. Energy Mater. 2015, 5.

(11) Li, Y.; Xu, L.; Liu, H.; Li, Y. Graphdiyne and Graphyne: From Theoretical Predictions to Practical Construction. Chem. Soc. Rev. 2014, 43, 2572.

(12) Evans, K. E.; Nkansah, M. A.; Hutcherson, I. J.; Rogers, S. C. Molecular Network 
Design. Nature 1991, 353, 124.

(13) Han, Z.; Lina, Z.; Dongshan, W.; Xinjian, Z.; Haofei, S. Effects of External Electric Field on Hydrogen Storage Performance of Li-Decorated Graphene Oxide. Chem. J. CHINESE Univ. 2016, 37, 100-107.

(14) Evans, K. E.; Alderson, A.; Christian, F. R. Auxetic Two-Dimensional Polymer Networks. An Example of Tailoring Geometry for Specific Mechanical Properties. $J$. Chem. Soc. Faraday Trans. 1995, 91, 2671.

(15) Baughman, R. H.; Galvão, D. S. Crystalline Networks with Unusual Predicted Mechanical and Thermal Properties. Nature 1993, 365, 735-737.

(16) Qin, R.; Zheng, J.; Zhu, W. Sign-Tunable Poisson’s Ratio in Semi-Fluorinated Graphene. Nanoscale 2017, 9, 128-133.

(17) Jiang, J.-W.; Chang, T.; Guo, X.; Park, H. S. Intrinsic Negative Poisson’s Ratio for Single-Layer Graphene. Nano Lett. 2016, 16, 5286-5290.

(18) Grima, J. N.; Grech, M. C.; Grima-Cornish, J. N.; Gatt, R.; Attard, D. Giant Auxetic Behaviour in Engineered Graphene. Ann. Phys. 2018.

(19) Grima-Cornish, J. N.; Grima, J. N.; Evans, K. E. On the Structural and Mechanical Properties of Poly(Phenylacetylene) Truss-Like Hexagonal Hierarchical Nanonetworks. Phys. Status Solidi Basic Res. 2017, 254.

(20) Degabriele, E. P.; Grima-Cornish, J. N.; Attard, D.; Caruana-Gauci, R.; Gatt, R.; Evans, K. E.; Grima, J. N. On the Mechanical Properties of Graphyne, Graphdiyne, and Other Poly(Phenylacetylene) Networks. Phys. status solidi 2017, 254.

(21) Trapani, L.; Gatt, R.; Mizzi, L.; Grima, J. N. Mechanical Properties of 2D Flexyne and Reflexyne Polyphenylacetylene Networks: A Comparative Computer Studies with Various Force-Fields. Task Q. 2015, 19, 237-296.

(22) Grima, J. N.; Attard, D.; Cassar, R. N.; Farrugia, L.; Trapani, L.; Gatt, R. On the Mechanical Properties and Auxetic Potential of Various Organic Networked Polymers. Mol. Simul. 2008, 34.

(23) Li, Y.; Li, Y. Two Dimensional Polymers-Progress of Full Carbon Graphyne. ACTA 
Polym. Sin. 2015,2, 147-165.

(24) Yang, L.; Harrysson, O.; West, H.; Cormier, D. Mechanical Properties of 3D ReEntrant Honeycomb Auxetic Structures Realized via Additive Manufacturing. Int. J. Solids Struct. 2015, 69-70, 475-490.

(25) Baughman, R. H.; Galvão, D. S.; Cui, C.; Wang, Y.; Tománek, D. Fullereneynes: A New Family of Porous Fullerenes. Chem. Phys. Lett. 1993, 204, 8-14.

(26) Baughman, R. H.; Galvão, D. S. Tubulanes: Carbon Phases Based on Cross-Linked Fullerene Tubules. Chem. Phys. Lett. 1993, 211, 110-118.

(27) Majidi, R. Electronic Properties of Porous Graphene, Alpha-Graphyne, Graphene-like, and Graphyne-like BN Sheets. Can. J. Phys. 2016, 94, 305-309.

(28) Majidi, R. Electronic Properties of Graphyne Nanotubes Filled with Small Fullerenes: A Density Functional Theory Study. J. Comput. Electron. 2016, 15 (4, SI), 1263-1268.

(29) Sebastiani, D.; Parker, M. A. Polyhedral Phenylacetylenes: The Interplay of Aromaticity and Antiaromaticity in Convex Graphyne Substructures. Symmetry-Basel. 2009, 1 (2), 226-239.

(30) Wang, J. T.; Chen, C.; Li, H. D.; Mizuseki, H.; Kawazoe, Y. Three-Dimensional Carbon Allotropes Comprising Phenyl Rings and Acetylenic Chains in Sp plus Sp(2) Hybrid Networks. Sci. Rep. 2016, 6.

(31) Grima, J. N.; Evans, K. E. Self Expanding Molecular Networks. Chem. Commun. 2000, 16 .

(32) Wojciechowski, K. W. Two-Dimensional Isotropic System with a Negative Poisson Ratio. Phys. Lett. A 1989, 137, 60-64.

(33) Wojciechowski, K. W. Non-Chiral, Molecular Model of Negative Poisson Ratio in Two Dimensions. J. Phys. A. Math. Gen. 2003, 36, 11765.

(34) Bilski, M.; Wojciechowski, K. W. Tailoring Poisson’s Ratio by Introducing Auxetic Layers. Phys. status solidi 2016, 253, 1318-1323.

(35) Dudek, M. R.; Grabiec, B.; Wojciechowski, K. W. Molecular Dynamics Simulations 
of Auxetic Ferrogel. Rev. Adv. Mater. Sci. 2007, 14, 167-173.

(36) Narojczyk, J. W.; Alderson, A.; Imre, A. R.; Scarpa, F.; Wojciechowski, K. W. Negative Poisson's Ratio Behavior in the Planar Model of Asymmetric Trimers at Zero Temperature. J. Non. Cryst. Solids 2008, 354, 4242-4248.

(37) Strek, T.; Jopek, H.; Wojciechowski, K. W. The Influence of Large Deformations on Mechanical Properties of Sinusoidal Ligament Structures. Smart Mater. Struct. 2016, 25.

(38) Tretiakov, K. V; Pigłowski, P. M.; Hyżorek, K.; Wojciechowski, K. W. Enhanced Auxeticity in Yukawa Systems Due to Introduction of Nanochannels in [001]Direction. Smart Mater. Struct. 2016, 25.

(39) Tretiakov, K. V; Wojciechowski, K. W. Poisson’s Ratio of Simple Planar “isotropic” Solids in Two Dimensions.’ Phys. status solidi 2007, 244, 1038-1046.

(40) Brańka, A. C.; Heyes, D. M.; Wojciechowski, K. W. Auxeticity of Cubic Materials. Phys. status solidi 2009, 246, 2063-2071.

(41) Grima, J. N.; Zammit, V.; Gatt, R.; Alderson, A.; Evans, K. E. Auxetic Behaviour from Rotating Semi-Rigid Units. Phys. Status Solidi Basic Res. 2007, 244.

(42) Grima, J. N.; Mizzi, L.; Azzopardi, K. M.; Gatt, R. Auxetic Perforated Mechanical Metamaterials with Randomly Oriented Cuts. Adv. Mater. 2016, 28.

(43) Lakes, R. Foam Structures with a Negative Poisson's Ratio. Science. 1987, 235, 10381041.

(44) Gatt, R.; Vella Wood, M.; Gatt, A.; Zarb, F.; Formosa, C.; Azzopardi, K. M.; Casha, A.; Agius, T. P.; Schembri-Wismayer, P.; Attard, L.; et al. Negative Poisson’s Ratios in Tendons: An Unexpected Mechanical Response. Acta Biomater. 2015, 24.

(45) Scarpa, F.; Panayiotou, P.; Tomlinson, G. Numerical and Experimental Uniaxial Loading on In-Plane Auxetic Honeycombs. J. Strain Anal. Eng. Des. 2000, 35, 383388.

(46) Scarpa, F.; Remillat, C.; Landi, F. P.; Tomlinson, G. R. Damping Modelization of Auxetic Foams. In SPIE's 7th Annual International Symposium on Smart Structures 
and Materials; 2000.

(47) Scarpa, F.; Pastorino, P.; Garelli, A.; Patsias, S.; Ruzzene, M. Auxetic Compliant Flexible PU Foams: Static and Dynamic Properties. Phys. status solidi 2005, 242, 681-694.

(48) Scarpa, F.; Smith, C. W.; Ruzzene, M.; Wadee, M. K. Mechanical Properties of Auxetic Tubular Truss-like Structures. Phys. Status Solidi Basic Res. 2008, 245, 584 590.

(49) Alderson, K. L.; Simkins, V. R.; Coenen, V. L.; Davies, P. J.; Alderson, A.; Evans, K. E. How to Make Auxetic Fibre Reinforced Composites. Phys. Status Solidi Basic Res. 2005.

(50) Grima, J. N.; Williams, J. J.; Evans, K. E. Networked Calix[4]Arene Polymers with Unusual Mechanical Properties. Chem. Commun. 2005, 32.

(51) Grima, J. N.; Caruana-Gauci, R. Mechanical Metamaterials: Materials That Push Back. Nat. Mater. 2012, 11 (7).

(52) Baughman, R. H.; Stafström, S.; Cui, C.; Dantas, S. O. Materials with Negative Compressibilities in One or More Dimensions. Science (80-. ). 1998, 279, 1522-1524.

(53) Grima, J. N.; Attard, D.; Caruana-Gauci, R.; Gatt, R. Negative Linear Compressibility of Hexagonal Honeycombs and Related Systems. Scr. Mater. 2011, 65.

(54) Fortes, A. D.; Suard, E.; Knight, K. S. Negative Linear Compressibility and Massive Anisotropic Thermal Expansion in Methanol Monohydrate. Science. 2011, 331 (6018), $742-746$.

(55) Cairns, A. B.; Thompson, A. L.; Tucker, M. G.; Haines, J.; Goodwin, A. L. Rational Design of Materials with Extreme Negative Compressibility: Selective Soft-Mode Frustration in $\mathrm{KMn}[\mathrm{Ag}(\mathrm{CN}) 2$ ] 3. J. Am. Chem. Soc. 2012, 134, 4454-4456.

(56) Li, W.; Probert, M. R.; Kosa, M.; Bennett, T. D.; Thirumurugan, A.; Burwood, R. P.; Parinello, M.; Howard, J. A. K.; Cheetham, A. K. Negative Linear Compressibility of a Metal-Organic Framework. J. Am. Chem. Soc. 2012, 134, 11940-11943.

(57) Coudert, F.-X. Systematic Investigation of the Mechanical Properties of Pure Silica 
Zeolites: Stiffness, Anisotropy, and Negative Linear Compressibility. Phys. Chem. Chem. Phys. 2013, 15 (38), 16012.

(58) Ortiz, A. U.; Boutin, A.; Fuchs, A. H.; Coudert, F. X. Anisotropic Elastic Properties of Flexible Metal-Organic Frameworks: How Soft Are Soft Porous Crystals? Phys. Rev. Lett. 2012, 109, 1-5.

(59) Cairns, A. B.; Goodwin, A. L. Negative Linear Compressibility. Phys. Chem. Chem. Phys. 2015, 17, 20449-20465.

(60) Serra-Crespo, P.; Dikhtiarenko, A.; Stavitski, E.; Juan-Alcañiz, J.; Kapteijn, F.; Coudert, F.-X.; Gascon, J. Experimental Evidence of Negative Linear Compressibility in the MIL-53 Metal-organic Framework Family. CrystEngComm 2015, 17, 276-280.

(61) Caruana-Gauci, R.; Degabriele, E. P.; Attard, D.; Grima, J. N. Auxetic Metamaterials Inspired from Wine-Racks. J. Mater. Sci. 2018, 53, 5079-5091.

(62) Pozniak, A. A.; Kaminski, H.; Kedziora, P.; Maruszewski, B.; Strek, T.; Wojciechowski, K. W. Anomalous Deformation of Constrained Auxetic Square. Rev. Adv. Mater. Sci. 2010, 23, 169-174.

(63) Strek, T.; Maruszewski, B. T.; Narojczyk, J. W.; Wojciechowski, K. W. Finite Element Analysis of Auxetic Plate Deformation. J. Non. Cryst. Solids 2008, 354, $4475-4480$

(64) Sun, H.; Mumby, S. J.; Maple, J. R.; Hagler, A. T. An Ab Initio CFF93 All-Atom Force Field for Polycarbonates. J. Am. Chem. Soc. 1994.

(65) Sun, H. Ab Initio Calculations and Force Field Development for Computer Simulation of Polysilanes. Macromolecules 1995, 28, 701-712.

(66) Ewald, P. P. Die Berechnung Optischer Und Elektrostatischer Gitterpotentiale. Ann. Phys. 1921, 369, 253-287.

(67) Masters, I. G.; Evans, K. E. Models for the Elastic Deformation of Honeycombs. Compos. Struct. 1996, 35, 403-422.

(68) Barnes, D. L.; Miller, W.; Evans, K. E.; Marmier, A. Modelling Negative Linear Compressibility in Tetragonal Beam Structures. Mech. Mater. 2012, 46, 123-128. 
(69) Zhou, X.; Zhang, L.; Zhang, H.; Liu, Q.; Ren, T. 3D Cellular Models with Negative Compressibility through the Wine-Rack-Type Mechanism. Phys. status solidi 2016, 253, 1977-1993.

(70) Grima, J. N.; Evans, K. E. Auxetic Behavior from Rotating Triangles. J. Mater. Sci. 2006, 41, 3193-3196.

(71) Ortiz, A. U.; Boutin, A.; Fuchs, A. H.; Coudert, F.-X. Metal-organic Frameworks with Wine-Rack Motif: What Determines Their Flexibility and Elastic Properties? J. Chem. Phys. 2013, 138, 174703.

(72) Tretiakov, K. V.; Bilski, M. M.; Wojciechowski, K. W. Maximum Poisson’s Ratios in Planar Isotropic Crystals of Binary Hard Disks at High Pressures. Phys. Status Solidi Basic Res. 2017, 254. 


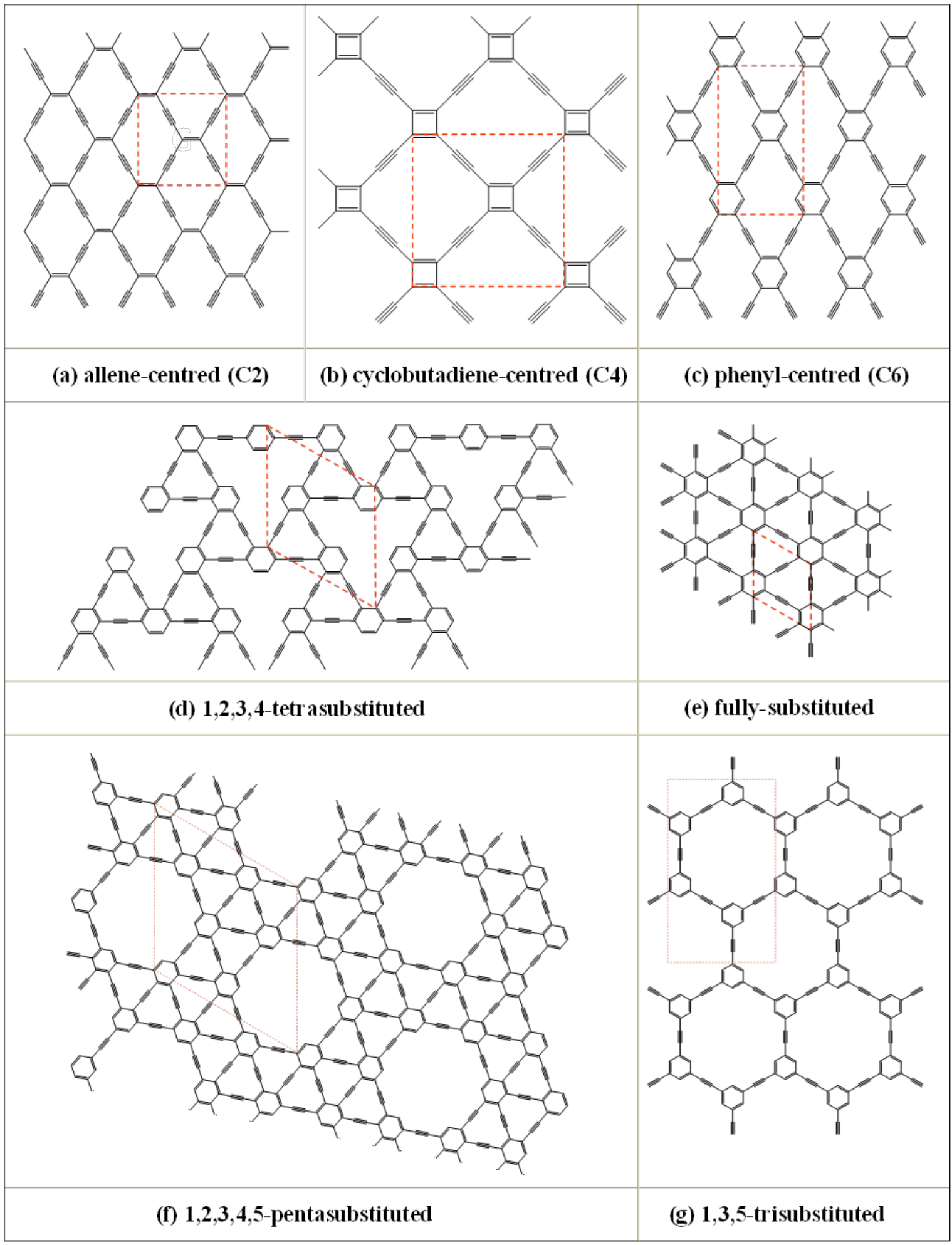

Figure 1: Schematic diagrams of the molecular-level systems studied. (a) and (b) are carbon allotropes simulated in this work which are meant to represent wine-rack like structures whilst (c) to (g) are poly(phenylacetylene) networks which are being analysed for their compressibility properties. Note that (a) have an allene centre, henceforth referred to as C2-n; (b) have a cyclobutadiene centre, henceforth referred to as C4- $n$, (c) 1,2,4,5 tetra-substituted phenyl centre, henceforth referred to as C6- $n$, (d) are 1,2,3,4 tetra-substituted referred to a polytriangles (e) are 1,2,3,4,5,6 fully-substituted referred to as graphyne networks (f) are 1,2,3,4,5 pentasubstituted referred to as mega-flexynes and (g) 1,3,5 tri-substituted referred to as flexynes. 


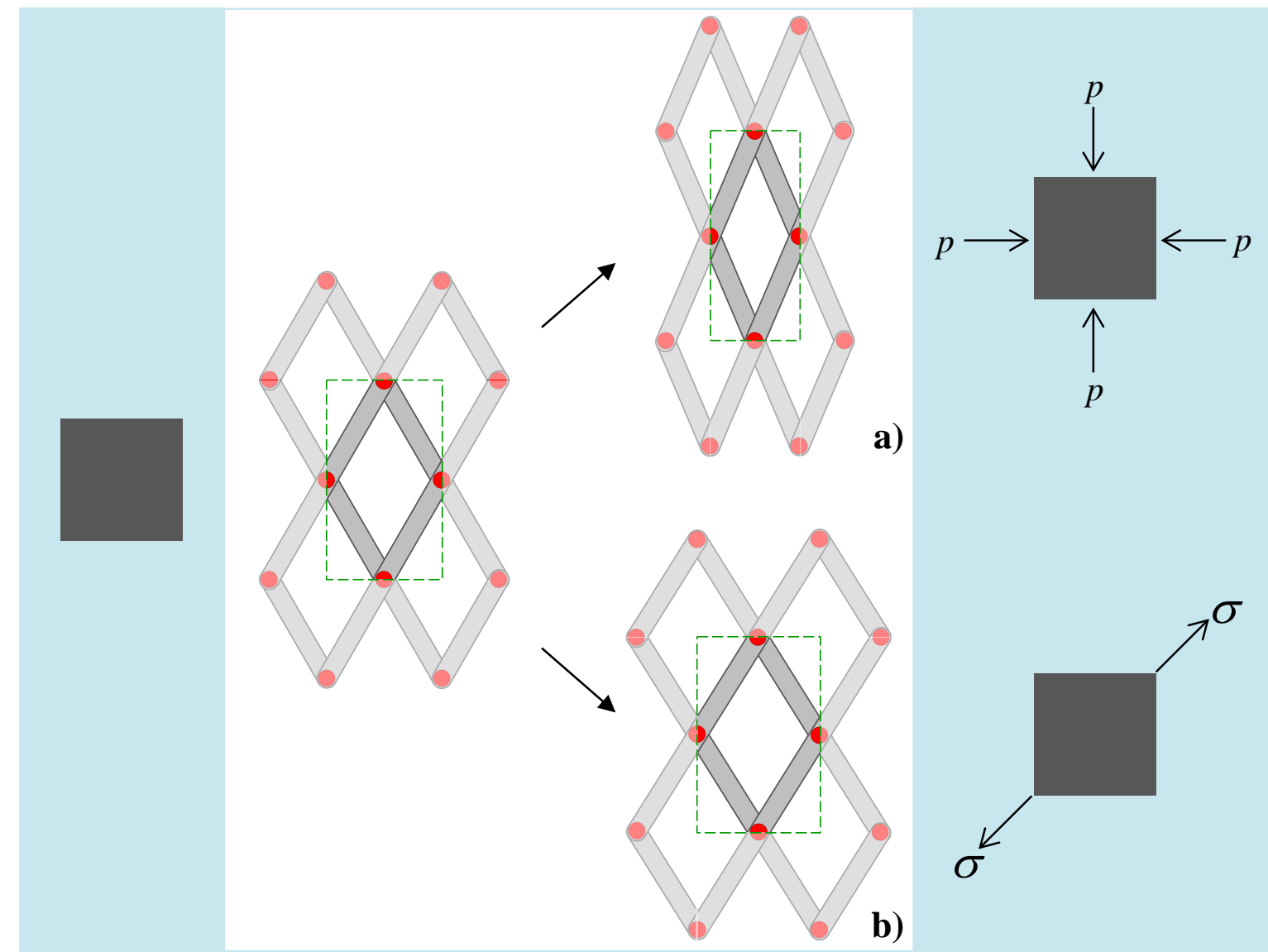

Figure 2: The idealised wine-rack model which can lead to (a) negative linear compressibility when subjected to hydrostatic pressure, $p$ or (b) negative Poisson's ratio when loaded off-axis by a uniaxial stress $\sigma$. 


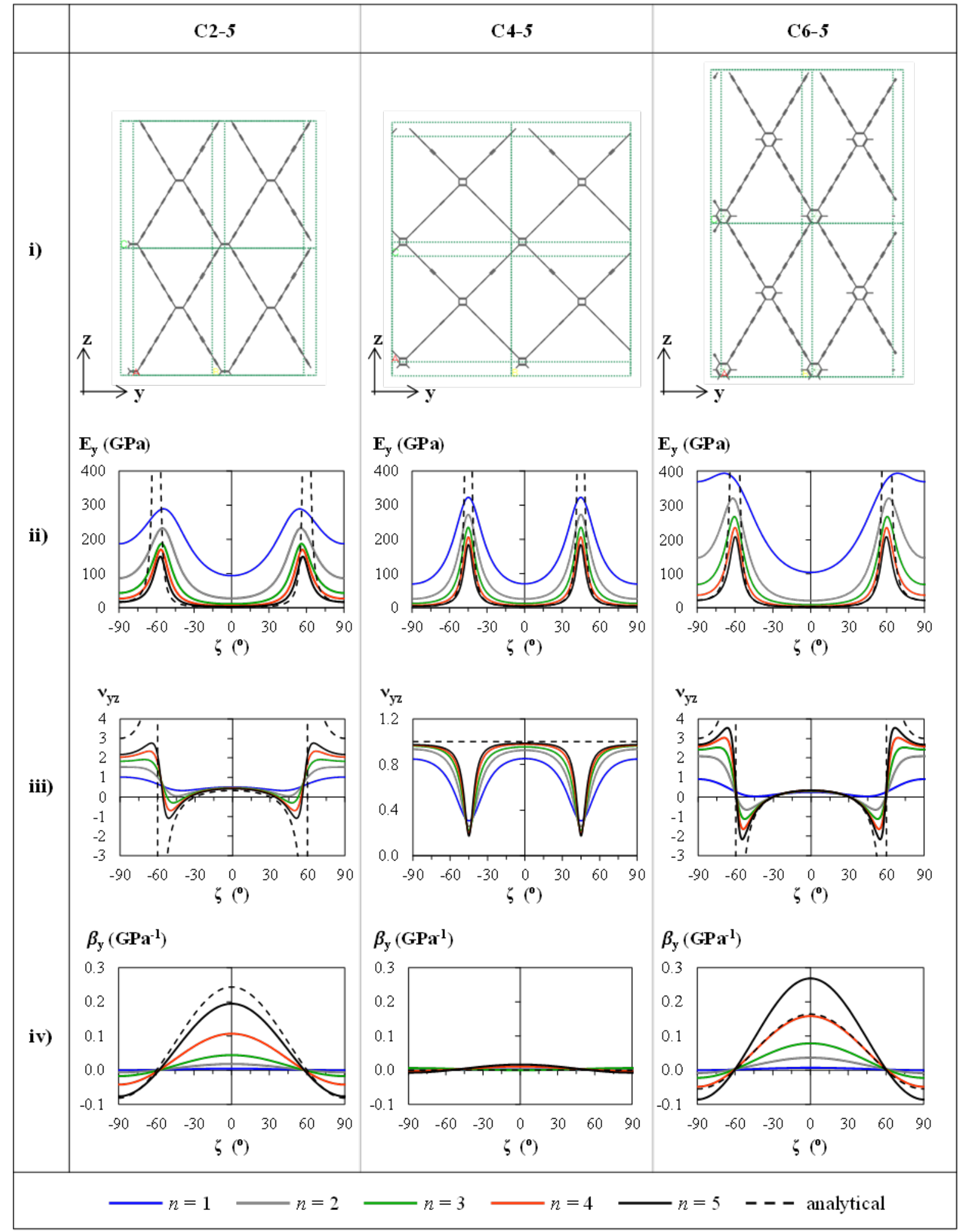

Figure 3: Results of the simulations and analysis for (a) the networks with the allene centre and (b) the networks with the cyclobutadiene centres in the (100) plane i.e. the plane of the networks, and where (i) show minimum energy conformations for systems at $p=0 \mathrm{GPa}$ with $n=5$; whilst (ii), (iii) and (iv) show the variation of the Young's moduli $\left(E_{y}\right)$; Poisson's ratio $\left(v_{y z}\right)$ and compressibility $\left(\beta_{y}\right)$ in the (100) yz-plane at an angle $\zeta$ with the $y$-direction. The equivalent data for 1,2,4,5 tetra-substituted networks, re-analysed from Degabriele et al. are 
shown in (c). Note that as illustrated in the legend the solid lines in the graph represent the data related to the molecular networks whilst the dotted lines relate to the prediction by the analytical model 


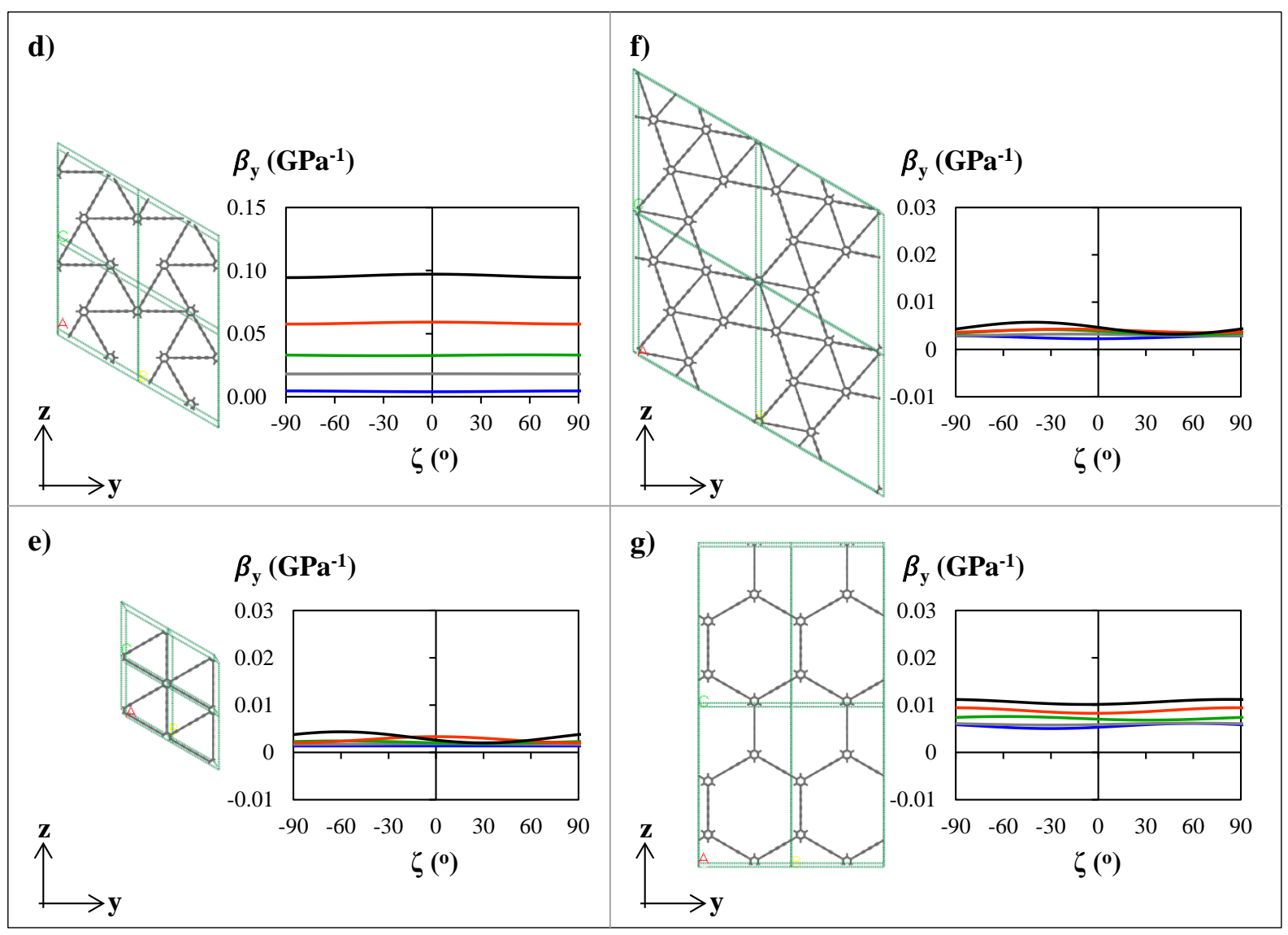

Figure 4: Compressibility of the poly(phenylacetylene) networks shown in Figure 2 (d) to (g) . Note that the compressibility plots for systems illustrated in (a) to (c) are illustrated in figure 3. Also, as shown in the legend the solid lines with different colours in the graphs represent 


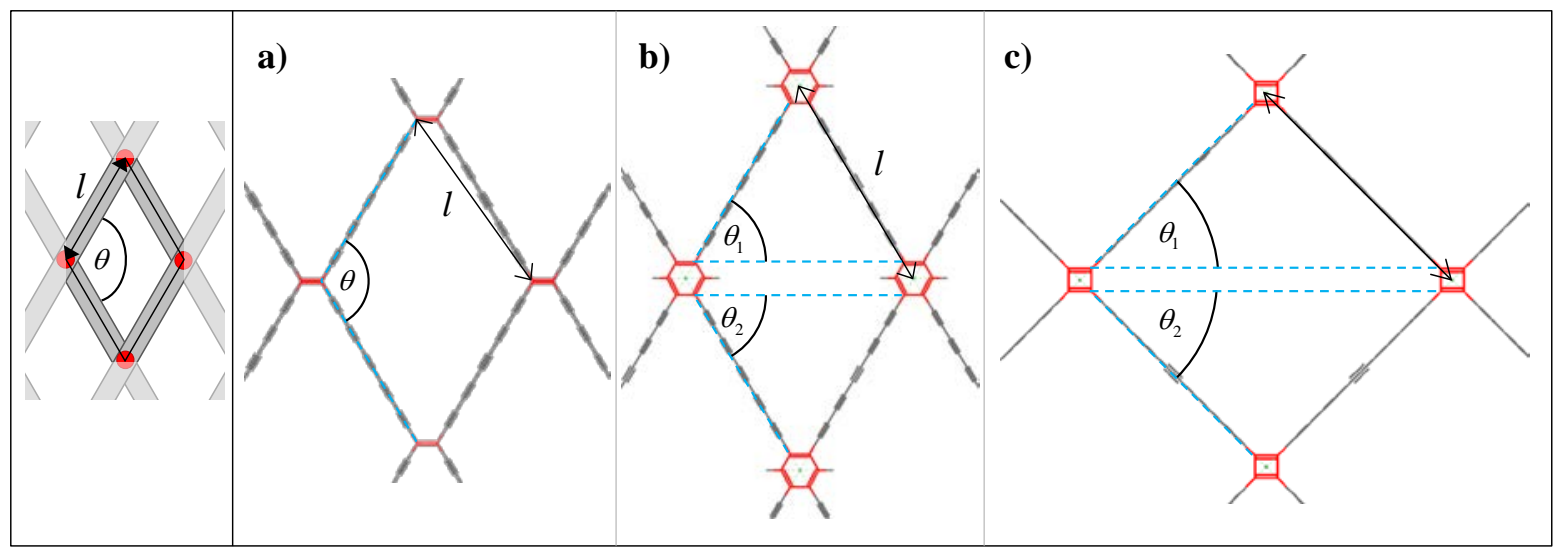

Figure 5: Schematic diagram which relates the idealised wine-rack model to the molecular systems studied here for systems with (a) an allene (b) a cyclobutadiene and (c) phenyl centre and a definition of how the in-plane length $(I)$ and angle measurements ( $\theta$ where for systems (b) and (c), $\theta=\theta_{1}+\theta_{2}$ ) were taken. Note the off-set between the acetylene chains and the hypothetical ligaments $(I)$ in the idealised model in the case of (a). 


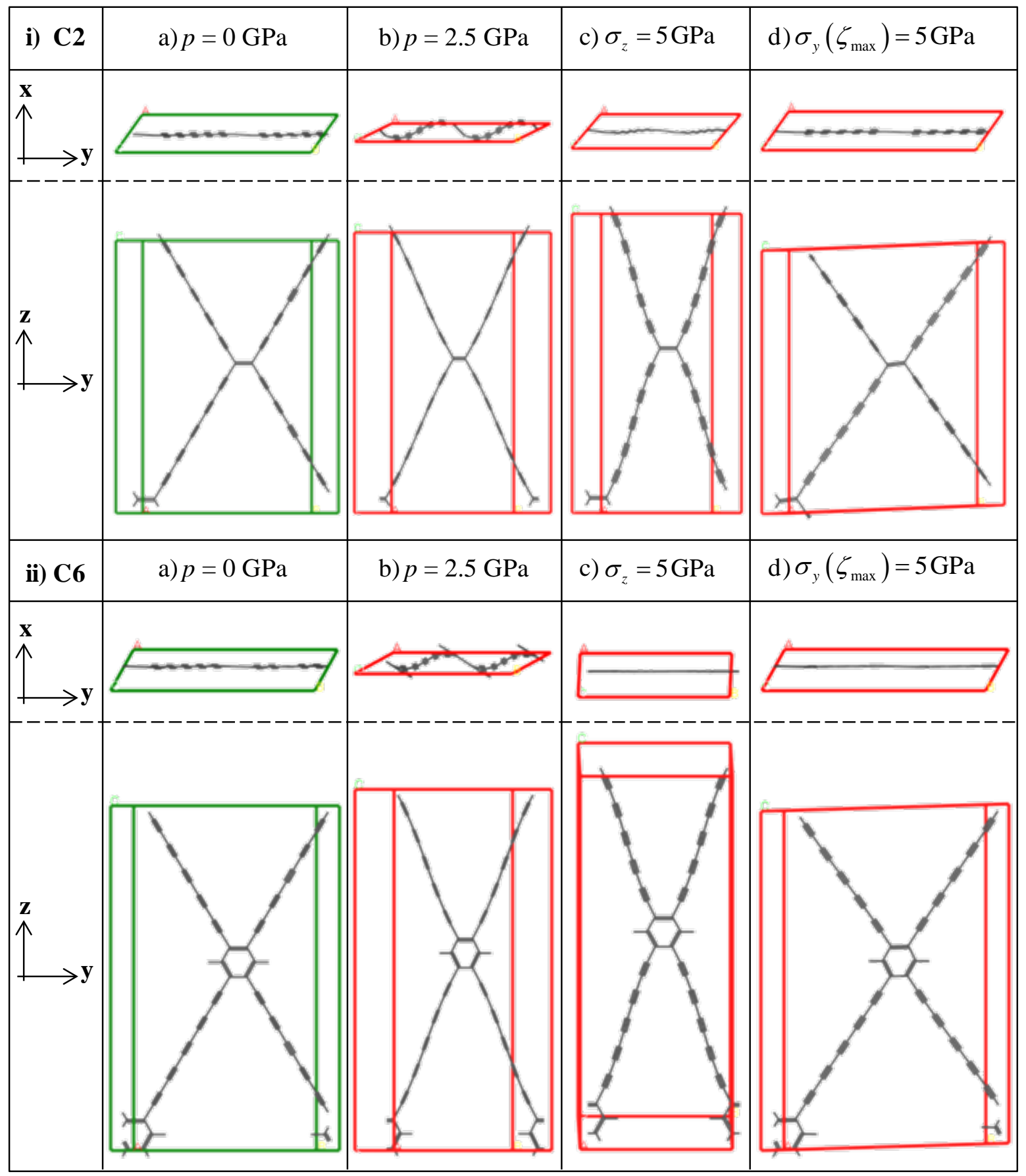

Figure 6: Images of the minimum energy conformation in the $y z$-plane for the systems (i) C2-5 and (ii) C6-5 at (a) hydrostatic pressure, $p=0 \mathrm{GPa}$; (b) $p=2.5 \mathrm{GPa}$; (c) uniaxial loading $\sigma_{z}=5 \mathrm{GPa}$ in [001] direction and (d) off-axis loading $\sigma_{y}\left(\zeta_{\max }\right)=5 \mathrm{GPa}$ in the direction of maximum auxeticity in the $y z$-plane which is at an angle $\zeta_{\max }$ to the $y$-direction. Note that the unit cell for the unloaded and loaded conformations are being shown in green and red respectively. 
Table 1: The value of $\zeta$ at maximum Young's modulus for all C2, C4 and C6 systems.

\begin{tabular}{|c|c|c|c|c|c|c|}
\hline & \multicolumn{2}{|c|}{ C2 system } & \multicolumn{2}{c|}{ C4 system } & \multicolumn{2}{c|}{ C6 system } \\
\hline $\boldsymbol{n}$ & $\zeta_{\max }\left({ }^{\circ}\right)$ & $E_{y z}^{\zeta}(\mathrm{GPa})$ & $\zeta_{\max }\left({ }^{\circ}\right)$ & $E_{y z}^{\zeta}(\mathrm{GPa})$ & $\zeta_{\max }\left({ }^{\circ}\right)$ & $E_{y z}^{\zeta}(\mathrm{GPa})$ \\
\hline $\mathbf{1}$ & 55.15 & 288.95 & 44.71 & 322.77 & 68.71 & 396.07 \\
\hline $\mathbf{2}$ & 55.37 & 232.66 & 45.05 & 272.60 & 61.79 & 322.34 \\
\hline $\mathbf{3}$ & 56.03 & 187.20 & 45.05 & 235.23 & 59.74 & 266.99 \\
\hline $\mathbf{4}$ & 56.69 & 170.16 & 44.94 & 206.82 & 60.07 & 236.23 \\
\hline $\mathbf{5}$ & 56.91 & 149.86 & 45.17 & 183.87 & 59.74 & 209.44 \\
\hline
\end{tabular}




\section{Supplementary Information}

\section{On the Compressibility Properties of the Wine-Rack like Carbon Allotropes and Related Poly(phenylacetylene) Systems}

Edera P. Degabriele ${ }^{1}$, Daphne Attard ${ }^{1}$, James N. Grima-Cornish ${ }^{2}$, Roberto Caruana-Gauci ${ }^{1}$, Ruben Gatt ${ }^{1}$, Kenneth E. Evans ${ }^{3}$ and Joseph N. Grima*1,2

${ }^{1}$ Metamaterials Unit, Faculty of Science, University of Malta, Msida MSD 2080, Malta. ${ }^{2}$ Department of Chemistry, Faculty of Science, University of Malta, Msida MSD 2080, Malta. ${ }^{3}$ College of Engineering, Mathematics and Physical Sciences, University of Exeter, Exeter EX4 4QF, UK.

*Corresponding Author.email: joseph.grima@um.edu.mt

Part 1: Full report of the mechanical properties

Images of each minimised structure together with their off-axis plots are shown in Figure S1-S3 where it is evident that the systems adopt a wine-rack like structure where C2 and C6 systems have $\theta=120^{\circ}$ and C4 systems have $\theta=90^{\circ}$. The procedure used here was outlined in the main text and in Degabriele et al. Phys Stat. Solidi (b) 2017. 


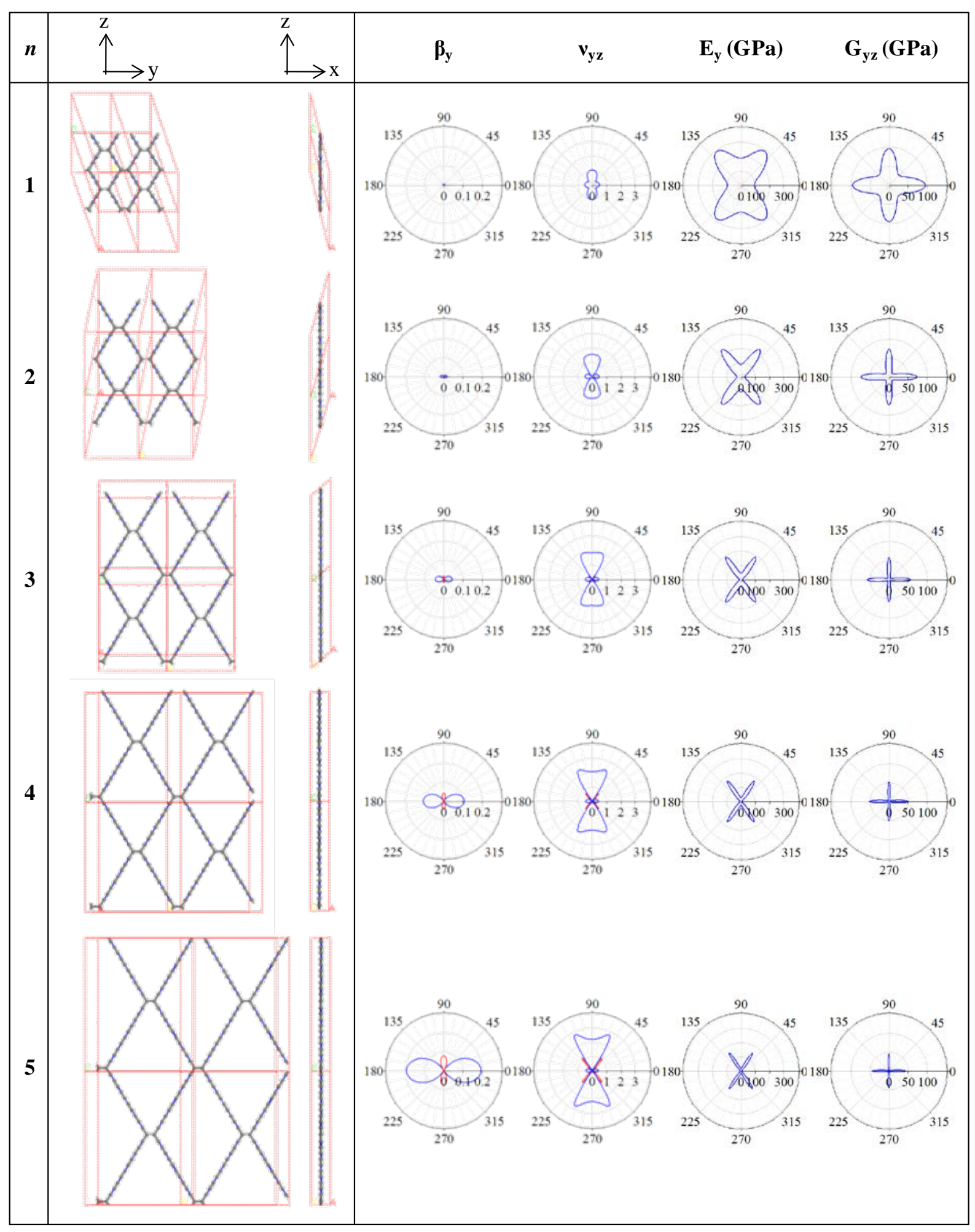

Figure S1: Images of the minimised C2- $n$ systems as simulated by the PCFF force-field where $n=$ $1,2 \ldots 5$ and their off-axis plots in the (100) plane ie. the plane which projects the wine-rack, of the compressibility $\beta_{\mathrm{y}}$, the Poisson's ratio $v_{\mathrm{yz}}$, Young's modulus $E_{\mathrm{y}}$ and shear modulus $G_{\mathrm{yz}}$. The red and blue lines show negative and positive regions respectively where it is clear that C2 systems show negative compressibility on-axis and negative Poisson's ratio off-axis (in the direction of the acetylene chains). 


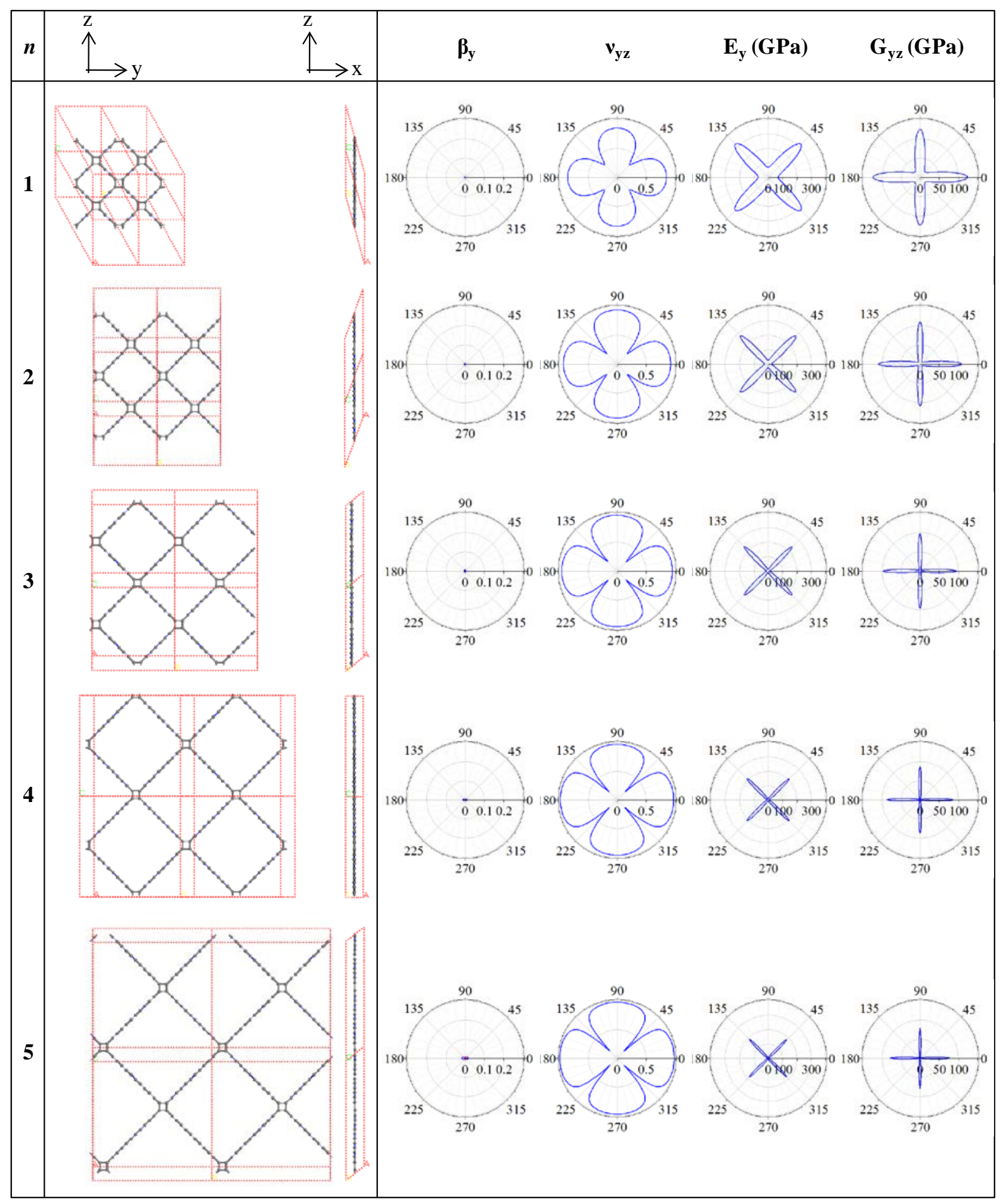

Figure S2: Images of the minimised C4- $n$ systems where $n=1,2 \ldots .5$ and their off-axis plots in the (100) plane of the compressibility $\beta_{\mathrm{y}}$, the Poisson's ratio $v_{\mathrm{yz}}$, Young's modulus $E_{\mathrm{y}}$ and shear modulus $G_{\mathrm{yz}}$ as simulated by the PCFF force-field. 


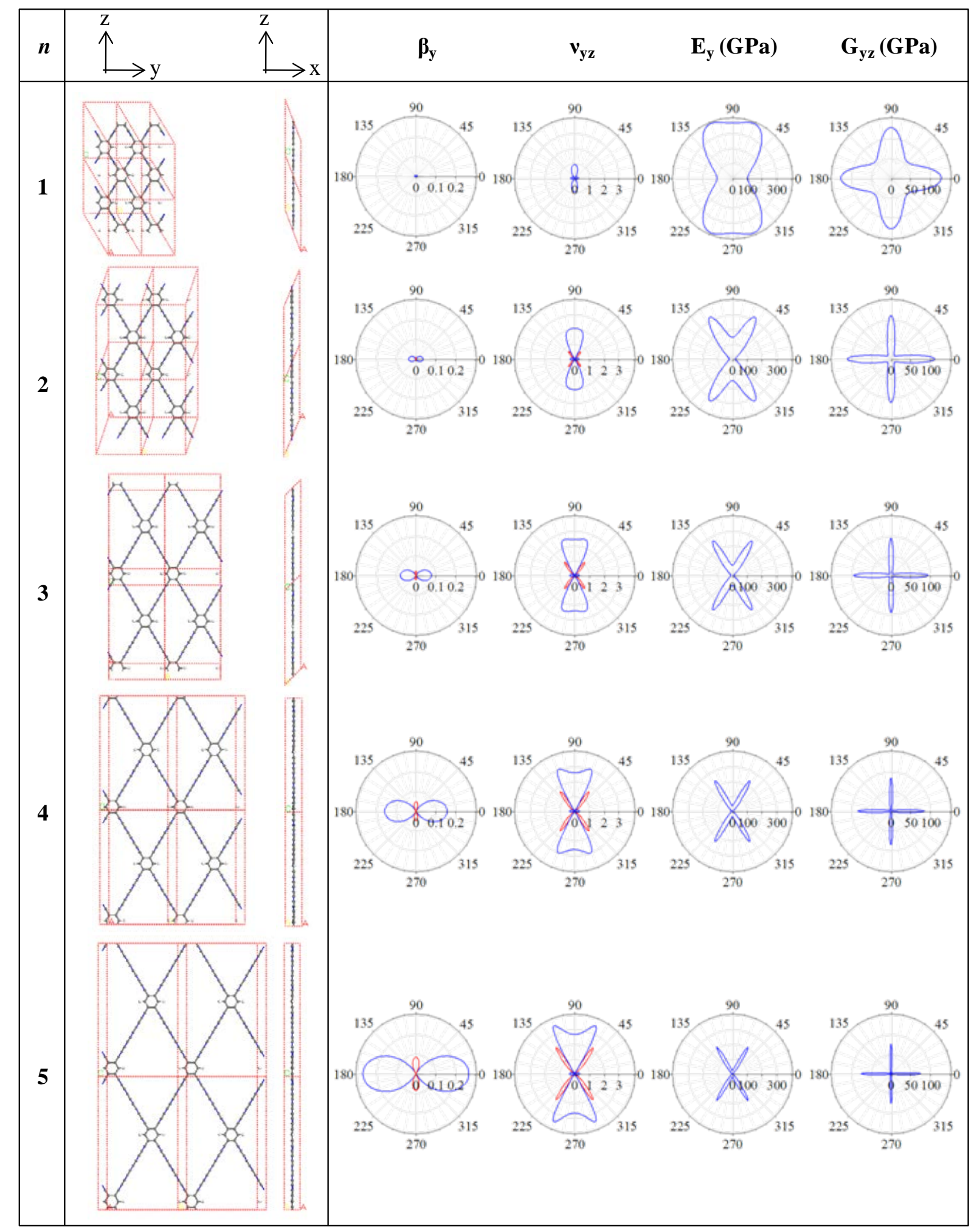

Figure S3: Images of the minimised C6- $n$ systems where $n=1,2 \ldots .5$ and their off-axis plots in the (100) plane of the compressibility $\beta_{\mathrm{y}}$, the Poisson's ratio $v_{\mathrm{yz}}$, Young's modulus $E_{\mathrm{y}}$ and shear modulus $G_{y z}$ as simulated by the PCFF force-field. 


\section{Part 2: Simulations of the nano-scale deformations when systems are subjected to pressure and} off-axis uniaxial stress.

In an attempt to assess the behaviour of such networks under uniaxial loading conditions and hydrostatic pressure conditions, two additional sets of simulations were performed on the C2-5 and C6-5 networks (see Figure S4), which homologues bear closest agreement with the idealised hinging wine-rack model. In the first set of simulations, the structures were subjected to a series of stresses applied in the $y z$-plane at an angle of $c .60^{\circ}$, at which auxeticity was found to be a maximum i.e. given the orientation of the cell and the direction of loading, the stress tensor takes the form:

$$
\boldsymbol{\sigma}=\frac{1}{4}\left(\begin{array}{ccc}
0 & 0 & 0 \\
0 & \sigma & -\sqrt{3} \sigma \\
0 & -\sqrt{3} \sigma & 3 \sigma
\end{array}\right)
$$

where:

$$
\sigma \in\{0,1,2, \ldots, 10\} \mathrm{GPa}
$$

In the second set of simulations, a series of hydrostatic pressures was applied to each of the structures, i.e. when the stress tensor takes the form of:

$$
\boldsymbol{\sigma}=\left(\begin{array}{lll}
p & 0 & 0 \\
0 & p & 0 \\
0 & 0 & p
\end{array}\right)
$$

where:

$$
p \in\{0.0,0.5,1.0,1.5, \ldots, 3.0\} \mathrm{GPa}
$$

For each of the off-axis and hydrostatic pressure simulations performed, the energy of the structure was minimised using the PCFF force-field and various measurements were taken (defined in Figure S4) which are reported in Figure S5 and S6.

\section{S2.1 Effect of uniaxial off-axis loading}

Figure S5-i) show the images of the deformed network superimposed over the corresponding undeformed network at zero stresses. A visual inspection of these images suggest that both C2-5 and C6-5 systems deform in a wine-rack like manner with the predominant changes occurring in the angle between the acetylene chain and the allene/phenyl central unit, equivalent to hinging in the idealised 
wine-rack model. This is further highlighted by the plots in Figure S5-iii) which show that $\theta_{1}$ increases and $\theta_{2}$ decreases, whilst the projection of the system in the $y$-and $z$-directions increases and decreases respectively, as expected for an idealised hinging wine-rack model. In addition to this, it is also evident that the distance between the two centroids which lies parallel to the direction of loading increases, while the length of the opposite chain decreases but to a smaller extent. Here it is interesting to note that the angle between the acetylene bonds making up the acetylene rib remains fairly constant. This suggests that deformation is not unimode but is a results of the interplay between hinging and stretching with hinging being the most dominant mechanism.

\section{S2.2 Effect of hydrostatic pressure}

The images in Figure S6-i) suggest that the systems deform mainly through a change in the angle between the acetylene chains leading to a contraction in the Y-direction and expansion in the lateral Z-direction and thus negative linear compressibility in the Z-direction. On further analysis of the acetylene chains, the plots suggest that the angle between the acetylene bonds changes while the length of the rib remains fairly constant upon deformation. This is shown pictorially in the image showing the $x y$-plane where the networks undergo flexure while the cell parameter $\gamma$ decreases upon deformation so that the projection in the $\mathrm{X}$-direction decreases. This suggests that the hinging mechanism which is responsible for the negative linear compressibility is also accompanied by flexure of the acetylene chains.

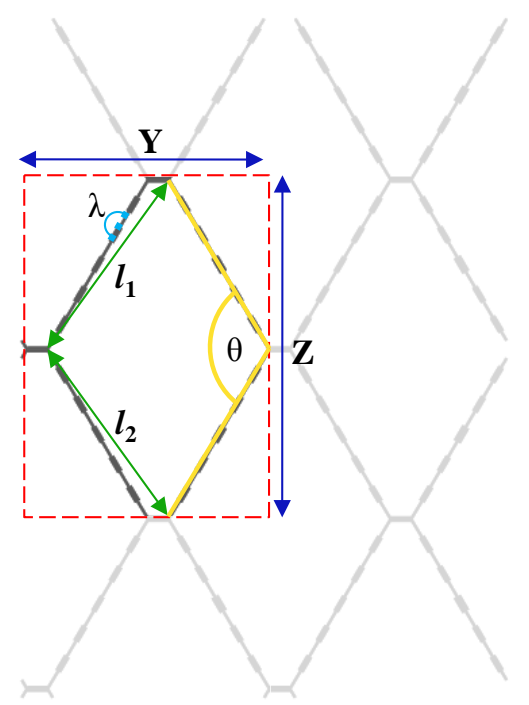

Figure S4: Image of the C2-5 system showing the cell parameters $\mathrm{Y}$ and $\mathrm{Z}$, the angle between the acetylene bonds $(\lambda)$, the length of the bonds making up the acetylene chain $\left(l_{\mathrm{a}}\right)$, the length from onecentroid to the other $\left(l_{1}\right.$ and $l_{2}$ ) and the angle between the ribs ( $\theta=\theta_{2}$ and $\theta_{1}=180-\theta_{2}$ ). 


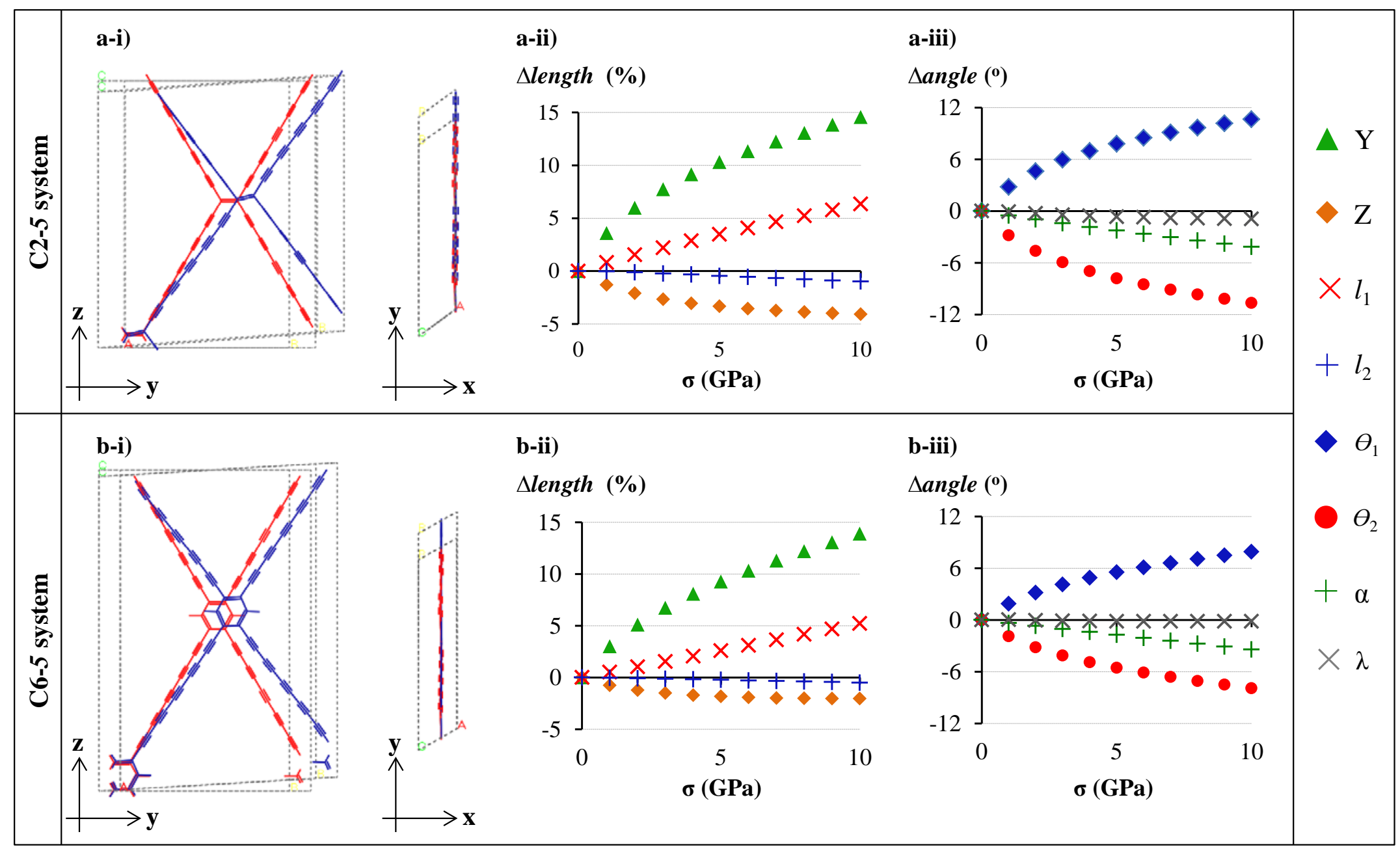

Figure S5: Super-imposed images of the undeformed (red) and deformed (blue) a-i) C2-5 system and b-i) C6-5 system in the (100) plane (the plane of the wine-rack) and in the (001) plane when subjected to off-axis loading in the direction of the ligaments. Plots of ii) the percentage change in length from one centroid to the other $\left(l_{1}\right.$ and $\left.l_{2}\right)$, the projection of the network in the $y$ - and $z$-directions (Y and Z) and iii) the change in the angles between the acetylene chains $\left(\theta_{1}\right.$ and $\left.\theta_{2}\right)$, the angle between the acetylene bonds making up the diagonal rib $(\lambda)$ and the cell parameter ( $\alpha$ ), against the applied stress when (a) C2-5 and (b) C6-5 are loaded at $\zeta=$ c. $60^{\circ}$ as simulated by the PCFF force-field. 


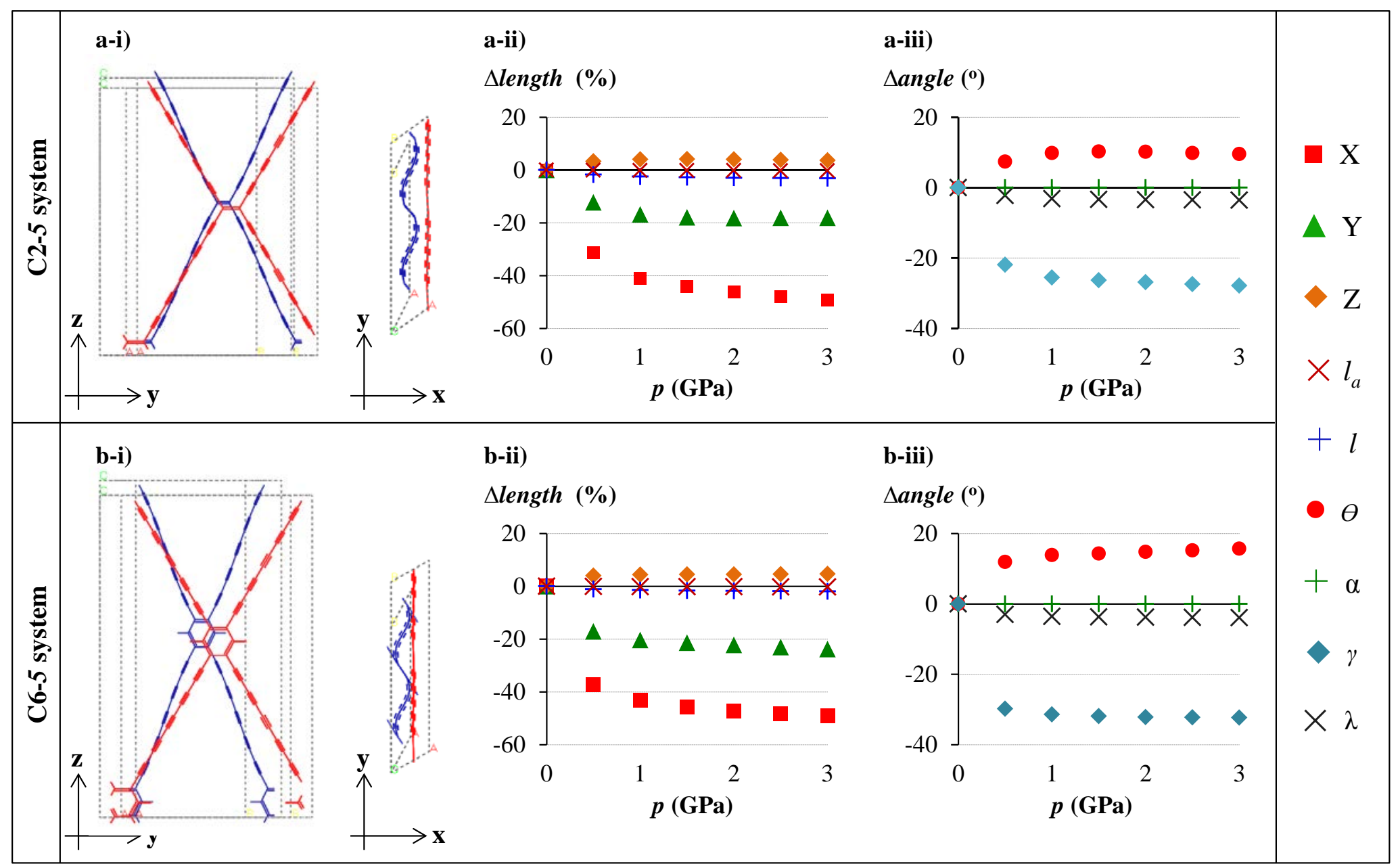

Figure S6: Super-imposed images of the undeformed (red) and deformed (blue) a-i) C2-5 system and b-i) C6-5 system in the (100) plane (the plane of the wine-rack) and in the (001) plane when subjected to hydrostatic pressure. Plots of ii) the percentage change in length from one centroid to the other $\left(l=l_{1}=\right.$ $l_{2}$ ), the projection of the network in the $x$-, y-and $z$-directions (X,Y and Z) and the length of the acetylene bond $\left(l_{\mathrm{a}}\right)$ and iii) the change in the angles between the acetylene chains $(\theta)$, the angle between the acetylene bonds making up the diagonal rib $(\lambda)$ and the cell parameters $\alpha$ and $\gamma$, against the applied hydrostatic pressure for (a) C2-5 and (b) C6-5 systems as simulated by the PCFF force-field. 